\author{
Cartas científicas \\ Rev Urug Cardiol 2021; 36: e705 \\ doi: $10.29277 /$ cardio.36.3.17

\section{Comunicación interauricular tipo seno venoso superior con retorno venoso pulmonar anómalo. Reporte de un caso}

\title{
Resumen
}

La comunicación interauricular tipo seno venoso superior es una cardiopatía congénita de baja prevalencia, más frecuente en mujeres, usualmente asociada con un retorno venoso pulmonar anómalo. La ecocardiografía transesofágica es el método diagnóstico más frecuente y debe complementarse con otros estudios de imagen como la angiotomografía de tórax o la resonancia magnética. Esta valoración no invasiva es fundamental para una correcta y completa caracterización anatómica con vistas a la resolución quirúrgica. La técnica quirúrgica de Warden resulta eficaz en aquellos pacientes donde el retorno venoso pulmonar anómalo desemboca en la vena cava superior por encima de la aurícula derecha, con baja incidencia de complicaciones y buenos resultados a largo plazo.

Palabras clave: COMUNICACIÓN INTERAURICULAR

SENO VENOSO SUPERIOR

TÉCNICA DE WARDEN

Sinus venosus atrial septal defect with abnormal pulmonary venous return. Case report.

\section{Summary}

Sinus venosus atrial septal defect is a low prevalence congenital heart disease, more frequently found in women, usually associated with abnormal pulmonary venous return. Transesophageal echocardiography is the most frequently used diagnostic method, and must be complemented with other imaging studies such as chest angiography or magnetic resonance. This assessment is essential to characterize the anatomical aspects that are essential for planning the corrective surgical strategy. In patients where the anomalous pulmonary venous return empties above the right atrium into the superior vena cava, the Warden surgical technique is effective, with a low incidence of complications and good longterm results.

Key words: $\quad$ ATRIAL SEPTAL DEFECT

SUPERIOR SINUS VENOSUS

WARDEN TECHNIQUE

\section{Defeito do septo atrial do seio venoso superior com retorno venoso pulmonar anormal. Relato de caso Resumo}

A comunicação interatrial do seio venoso superior é uma cardiopatia congênita de baixa prevalência, mais frequente em mulheres, com alto percentual de retorno venoso pulmonar anormal. A ecocardiografia transesofágica é o método diagnóstico mais frequente e deve ser complementada com outros exames de imagem, como angiografia de tórax ou ressonância magnética. Essa avaliação é essencial para analisar aspectos anatômicos essenciais para o planejamento da estratégia cirúrgica corretiva. Naqueles pacientes em que o retorno venoso pulmonar anômalo deságua acima do átrio direito na veia cava superior, a técnica cirúrgica de Warden é eficaz, com baixa incidência de complicações e bons resultados em longo prazo.

Palavras chave: COMUNICAÇÃO ENTRE ATRIOS

SEIO VENOSO SUPERIOR

TÉCNICA DE WARDEN

Sr. Editor:

\section{Introducción}

La comunicación interauricular (CIA) es la cardiopatía congénita no cianótica más frecuente en la edad adulta, con predominio en el sexo femenino (relación 2:1). La paciente que presentamos a continuación padece un defecto de tipo seno venoso superior, fenotipo de CIA con baja prevalen- cia $(<5 \%)$, que se asocia con anormalidades del retorno venoso pulmonar en un porcentaje mayor del $90 \%$.

El principal estudio para el diagnóstico de esta patología es la ecocardiografía. Es fundamental la angiotomografía de tórax para la valoración del retorno venoso anómalo en vistas a completar el diagnóstico y elaborar la estrategia terapéutica adecuada para cada caso. La corrección quirúrgica mediante la técnica de Warden ha mostrado 


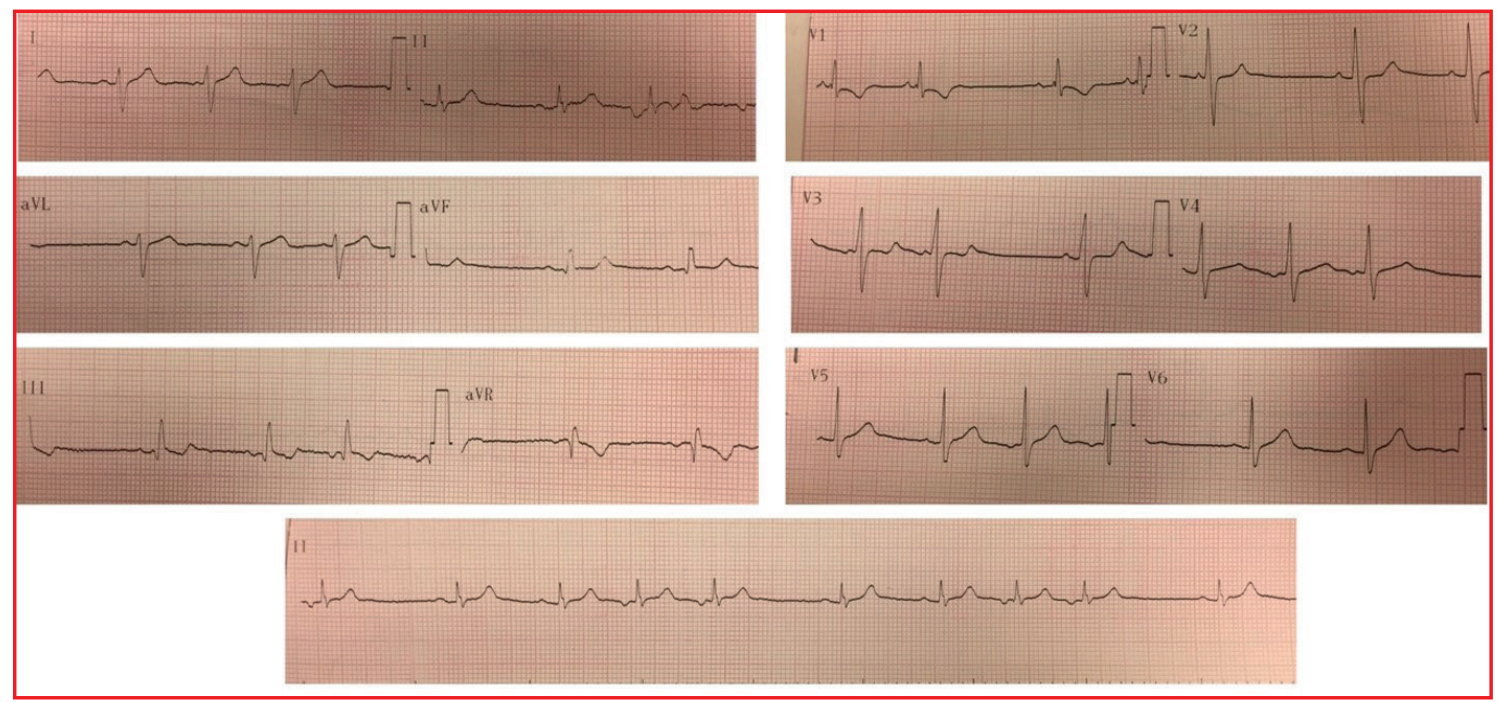

Figura 1. Electrocardiograma. Se observa un ritmo sinusal de $80 \mathrm{cpm}$ que alterna con un ritmo auricular bajo, QRS fino, eje eléctrico medio desviado a derecha (+120 grados), bloqueo incompleto de rama derecha.

excelentes resultados en aquellos pacientes donde el retorno venoso pulmonar anómalo desemboca en la vena cava superior (VCS), cobrando mayor relevancia cuanto más alto es este respecto a la aurícula derecha $(\mathrm{AD})$, ya que disminuye el riesgo de estenosis residual de la VCS y estenosis del retorno pulmonar corregido hacia la AI.

El propósito del presente artículo es comentar un caso clínico de esta patología de baja prevalencia, su forma de presentación, diagnóstico, tratamiento quirúrgico y evolución.

\section{Caso clínico}

Paciente de sexo femenino, 22 años, sin antecedentes personales a destacar. Consulta en policlínica por cuadro de disnea de esfuerzo (DE) clase funcional II de la New York Heart Association de 6 años de evolución, acompañado de palpitaciones esporádicas. Niega otros elementos patológicos de la esfera cardiovascular. En el examen físico cardiovascular destaca un ritmo regular de 75 ciclos por minuto (cpm), primer ruido normal, desdoblamiento fijo del segundo ruido, soplo sistólico eyectivo a nivel precordial con máxima auscultación en región paraesternal izquierda. Resto del examen físico normal. Electrocardiograma (ECG): ritmo sinusal de $80 \mathrm{cpm}$ que alterna con un ritmo auricular bajo, QRS fino, eje eléctrico medio desviado a derecha (+ 120 grados), bloqueo incompleto de rama derecha (BIRD) (figura 1). En la radiografía de tórax ( $\mathrm{Rx} T \mathrm{~T})$ de frente y perfil se observa a nivel de los campos pleuropulmonares una plétora pulmonar y a nivel mediastinal una silueta cardio-aorto-pericárdica aumentada, a expensas de aurícula y ventrículo derecho (VD), con un arco pulmonar prominente (figura 2). El ecocardiograma transesofágico reportó un ventrículo izquierdo de dimensiones normales, con función sistólica conservada, aurícula derecha (AD) levemente dilatada, VD severamente dilatado (diámetro $43 \mathrm{~mm}$ ) con función sistólica conservada, CIA tipo seno venoso superior de $18 \mathrm{~mm}$ de diámetro mayor con pasaje de flujo de izquierda a derecha que asocia retorno anómalo de vena pulmonar superior derecha (VPSD) a la VCS, $10 \mathrm{~mm}$ por encima de su desembocadura en la aurícula derecha, con una relación gasto cardíaco-pulmonar/gasto cardíaco-sistémico de 2,8 (Qp/Qs). Hipertensión arterial pulmonar moderada (presión sistólica de la arteria pulmonar $48 \mathrm{mmHg}$ ). Dado este último hallazgo, se solicita cateterismo cardíaco derecho que descarta hipertensión arterial pulmonar, informa CIA tipo seno venoso superior, con resistencias arteriales pulmonares (RVP) y sistémicas (RVS) normales con Qp/Qs de 3,13. Se realiza angiotomografía de tórax que evidencia retorno anómalo de VPSD que desemboca en VCS a unos 20 $\mathrm{mm}$ por encima de la $\mathrm{AD}$ (figura 3 ).

Dado el diagnóstico de CIA con elementos de sobrecarga de volumen en el VD y sin hipertensión pulmonar, se decide proceder al cierre del defecto septal interauricular y corrección del retorno venoso pulmonar anómalo mediante procedimiento de Warden. La paciente presentó una buena evolución posoperatoria y se le otorgó el alta hospitalaria a los 6 días, con un ETT que no evidenciaba shunt residual a través del septum interauricular. 


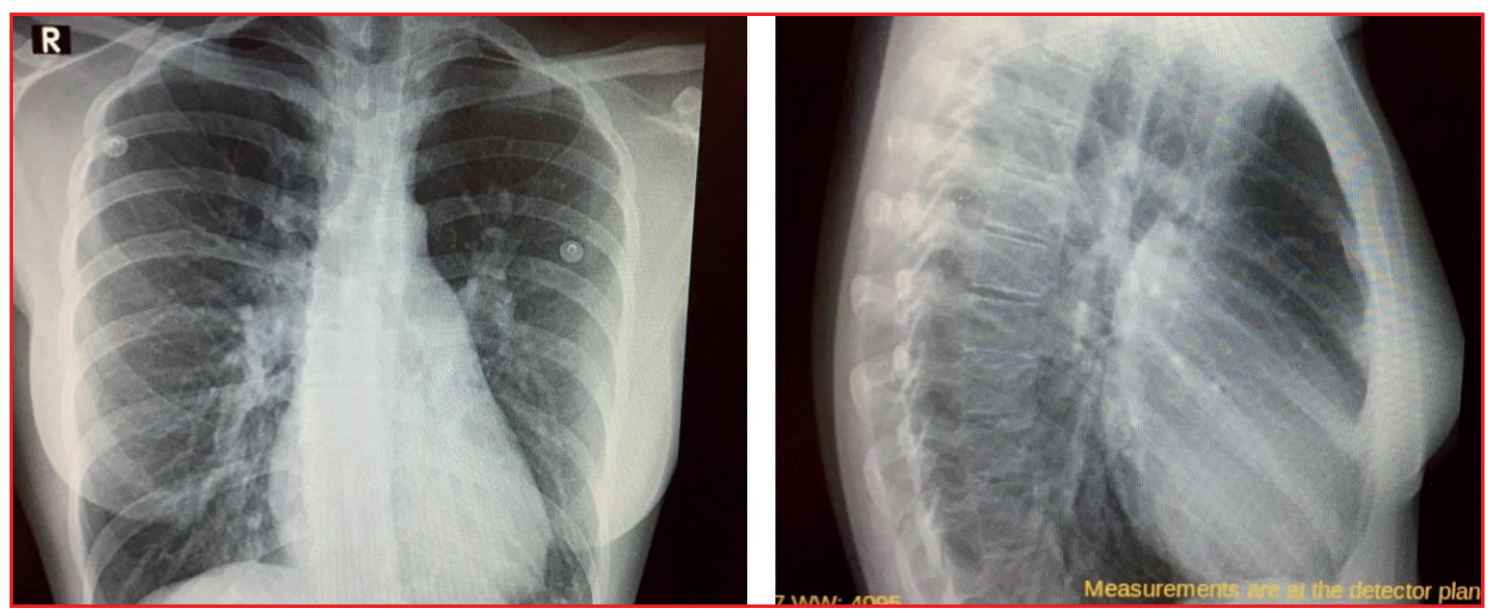

Figura 2. Radiografía de tórax frente y perfil. A nivel de campos pleuropulmonares se observa una plétora pulmonar y a nivel mediastinal una silueta cardioaortopericárdica aumentada, a expensas de aurícula y ventrículo derecho, con un arco pulmonar prominente.

\section{Discusión}

La CIA se clasifica en cinco variantes: tipo ostium secundum (la más prevalente, $80 \%$ ), ostium primum $(15 \%)$, seno venoso superior $(5 \%)$, seno venoso inferior $(<1 \%)$ y de tipo seno coronario $(<1 \%)^{(1)}$.

La paciente reportada presenta un defecto de tipo seno venoso superior, que se localiza inmediatamente por debajo de la desembocadura de la VCS. Desde el punto de vista embriológico se debe a la reabsorción incompleta del seno venoso en la $\mathrm{AD}$ o del crecimiento insuficiente del septum secundum. En el $95 \%$ de los casos se asocia con anormalidades del retorno venoso pulmonar, pudiendo desembocar este a nivel de la VCS o en la AD. Dicha cardiopatía congénita puede asociarse con otras alteraciones cardiovasculares, como la presencia de vena cava superior izquierda (5\%), estenosis de arteria pulmonar leve-moderada (4\%), comunicación interventricular pequeña $(2 \%)$, así como insuficiencia mitral, insuficiencia tricuspídea o cleft mitral ${ }^{(2)}$.

En esta patología, el shunt está dado tanto por el pasaje de sangre desde el retorno pulmonar anómalo a las cavidades derechas, así como a través de la propia CIA. La magnitud del shunt a través de esta última depende del gradiente de presión entre $\mathrm{AI}$ y $\mathrm{AD}$, compliance de ambos ventrículos, relación entre RVP y RVS y la presencia de otras cardiopatías asociadas (valvulopatía mitral-aórtica, cardiopatía isquémica, hipertensión arterial). En general, los pacientes cursan asintomáticos hasta la edad adulta; el $50 \%$ presentará síntomas a los 20 años, debido principalmente por disnea de esfuerzo y palpitaciones, siendo menos frecuente la falla cardíaca derecha y la infección pulmonar $^{(1)}$. Con respecto al examen físico, se destaca a nivel cardiovascular un soplo sistólico

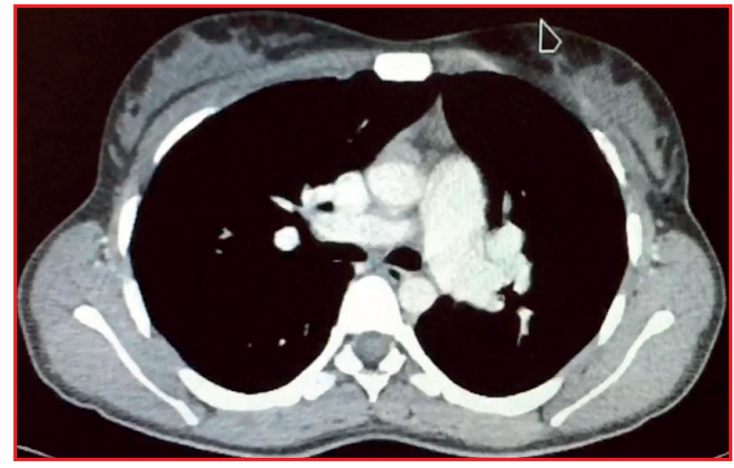

Figura 3. Angiotomografía de tórax con contraste. Se observa la vena pulmonar superior derecha (VPSD) desembocando a nivel de la vena cava superior (VCS).

eyectivo en foco pulmonar debido a hiperflujo y un desdoblamiento fijo del segundo ruido por aumento del tiempo diastólico del VD. Por su parte, los hallazgos electrocardiográficos son concordantes con la presencia del defecto interauricular (ritmo auricular bajo, desviación a derecha del EEM y BIRD). A nivel de la RxTx encontramos claros elementos de remodelado de cavidades derechas, así como signos de hiperflujo pulmonar. El ecocardiograma transtorácico es la herramienta fundamental para definir el diagnóstico y la estrategia terapéutica, permite valorar, entre otros, el tipo, el tamaño, la forma y la relación del defecto con estructuras adyacentes. El hallazgo de un VD severamente dilatado, en ausencia de valvulopatías significativas y patología pulmonar preexistente nos debe alertar de la presencia de un posible shunt interauricular. En este caso, el ecocardiograma transesofágico permitió identificar una CIA tipo seno venoso superior y la presencia de retorno anómalo de vena pulmonar superior derecha 
desembocando en la VCS. La valoración completa del retorno venoso anómalo debe complementarse con otros estudios de imagen como la angiotomografía de tórax con contraste o la resonancia magnética, donde es fundamental definir, entre otros valores, la distancia entre su desembocadura en la VCS y la AD, para planificar una correcta estrategia terapéutica ${ }^{(1)}$.

Si bien la presencia de hipertensión arterial pulmonar severa es poco frecuente, en la CIA $(<5 \%)$ el cateterismo cardíaco derecho se encuentra indicado cuando se presentan signos no invasivos de elevación de la presión arterial pulmonar (PSAP > $40 \mathrm{mmHg}$ ), siendo indicación clase $\mathrm{I}^{(1)}$. Nuestra paciente tiene indicación de cirugía cardíaca por presentar una CIA que asocia retorno venoso pulmonar anómalo. Dicha cirugía estuvo dirigida a lograr el cierre del defecto del septum interatrial y la corrección de dicho retorno anómalo. Existen varias técnicas quirúrgicas para la reparación. En la mayoría de los pacientes, el retorno anómalo desemboca a nivel de la $\mathrm{AD}$ o en la unión entre la VCS y esta última. En dicho caso, generalmente se procede al cierre del defecto septal mediante la sutura de un parche de pericardio autólogo o bovino, logrando el redireccionamiento del flujo de la vena pulmonar a la AI y eliminando el shunt izquierda-derecha. Sin embargo, a medida que la desembocadura es más cefálica, la utilización de este procedimiento podría generar ciertas complicaciones como la estenosis residual de la VCS y estenosis del retorno pulmonar corregido hacia la $\mathrm{AI}$, lo que conlleva consecuencias deletéreas tanto clínicas como en el pronóstico del paciente. Para ello existen dos alternativas con buenos resultados: la técnica del "doble parche" y la técnica de Warden. La primera consiste en realizar el cierre del defecto interauricular con ampliación de la VCS en su desembocadura en la AD. La segunda técnica, descrita por el cirujano cardiotorácico Hebert Warden en 1984 y llevada a cabo en nuestra paciente, consiste en transectar la VCS por encima de la vena pulmonar anómala y anastomosarla a la orejuela derecha, seguido del cierre del defecto interatrial mediante un parche de pericardio ${ }^{(3)}$. Varios estudios han comparado los resultados de estas técnicas y no se observan diferencias significativas en cuanto a mortalidad o estenosis de VCS, si bien esta última fue más prevalente en pacientes sometidos a la técnica de parche simple y doble parche. Por otra parte, se observó una mayor incidencia de disfunción del nodo sinusal con el uso del doble parche en relación con las otras dos, posiblemente relacionada con el daño de la arteria del nodo sinusal durante la incisión de la $\mathrm{AD}^{(4,5)}$.

Existen muy pocos estudios de seguimiento a largo plazo en pacientes con esta patología que son sometidos a procedimiento quirúrgico correctivo. Uno de ellos involucró el seguimiento de 105 pacientes a lo largo de 12 años, observando mejoría sintomática en un alto porcentaje (77\%), así como una baja incidencia de disfunción sinusal (6\%) y fibrilación auricular $(15 \%)^{(6)}$.

\section{Conclusión}

Los pacientes con CIA y retorno venoso anómalo pulmonar deben ser valorados en detalle mediante técnicas de imagen específicas, para llevar a cabo la técnica quirúrgica más adecuada en cada caso individual, evitando de esta forma el desarrollo de complicaciones que condicionen la morbilidad y posterior calidad de vida. La técnica de Warden resulta eficaz, con baja incidencia de complicaciones y buenos resultados a largo plazo.

Maximiliano Hernandez, Martín Antelo, Juan José Paganini, Martín Chappe, Víctor Dayan, Daniel Brusich Centro Cardiovascular Universitario. Departamento de Cirugía Cardíaca, Hospital de Clínicas. Correspondencia: Dr. Maximiliano Hernández. Correo electrónico: maximilianohernandezh@gmail.com

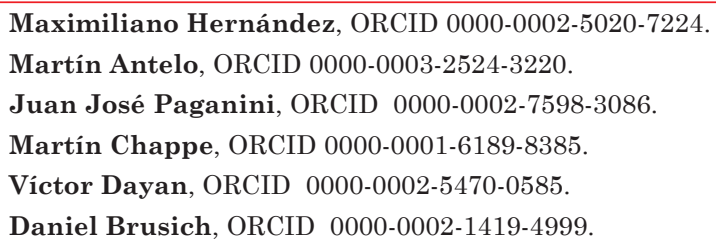




\section{Bibliografía}

1. Baumgartner H, De Backer J, Babu-Narayan SV, Budts W, Chessa M, Diller GP, et al. 2020 ESC Guidelines for the management of adult congenital heart disease. Eur Heart J. 2021;42(6):563645. doi: 10.1093/eurheartj/ehaa554.

2. Kirklin J, Blakstone E, Kirklin/Barratt-Boyes. Congenital Heart Disease. Cardiac Surgery: morphology, diagnostic criteria, natural history, techniques, results, and indications. 4th ed. Philadelphia:Elsevier;2013:1149-78

3. Warden HE, Gustafson RA, Tarnay TJ, Neal WA. An alternative method for repair of partial anomalous pulmonary venous connection to the superior vena cava. Ann Thorac Surg. 1984;38(6):6015. doi: 10.1016/s0003-4975(10)62317-x.
4. Stewart RD, Bailliard F, Kelle AM, Backer CL, Young L, Mavroudis C. Evolving surgical strategy for sinus venosus atrial septal defect: effect on sinus node function and late venous obstruction. Ann Thorac Surg. 2007;84(5):1651-5; discussion 1655. doi: 10.1016/j.athoracsur.2007.04.130.

5. Shahriari A, Rodefeld MD, Turrentine MW, Brown JW. Caval division technique for sinus venosus atrial septal defect with partial anomalous pulmonary venous connection. Ann Thorac Surg. 2006;81(1):224-9; discussion 229-30. doi: 10.1016/j. athoracsur.2005.07.015.

6. Attenhofer Jost CH, Connolly HM, Danielson GK, Bailey KR, Schaff HV, Shen WK, et al. Sinus venosus atrial septal defect: long-term postoperative outcome for 115 patients. Circulation. 2005;112(13):1953-8. doi:10.1161/CIRCULATIONAHA.104.493775. 